\title{
A SVDD and K-Means Based Early Warning Method for Dual-Rotor Equipment under Time-Varying Operating Conditions
}

\author{
Zhinong Jiang $\left(\mathbb{D}\right.$, Minghui Hu, Kun Feng $\mathbb{D}^{\mathbb{D}}$, and Hao Wang \\ College of Mechanical and Electrical Engineering, Beijing University of Chemical Technology, Beijing, China \\ Correspondence should be addressed to Kun Feng; kunfengphd@163.com
}

Received 15 August 2017; Revised 4 December 2017; Accepted 12 December 2017; Published 4 January 2018

Academic Editor: Sandris Ručevskis

Copyright (C) 2018 Zhinong Jiang et al. This is an open access article distributed under the Creative Commons Attribution License, which permits unrestricted use, distribution, and reproduction in any medium, provided the original work is properly cited.

Under frequently time-varying operating conditions, equipment with dual rotors like gas turbines is influenced by two rotors with different rotating speeds. Alarm methods of fixed threshold are unable to consider the influences of time-varying operating conditions. Hence, those methods are not suitable for monitoring dual-rotor equipment. An early warning method for dual-rotor equipment under time-varying operating conditions is proposed in this paper. The influences of time-varying rotating speeds of dual rotors on alarm thresholds have been considered. Firstly, the operating conditions are divided into several limited intervals according to rotating speeds of dual rotors. Secondly, the train data within each interval is processed by SVDD and the allowable ranges (i.e., the alarm threshold) of the vibration are determined. The alarm threshold of each interval of operating conditions is obtained. The alarm threshold can be expressed as a sphere, whose controlling parameters are the coordinate of the center and the radius. Then, the cluster center of the test data, whose alarm state is to be judged, can be extracted through $K$-means. Finally, the alarm state can be obtained by comparing the cluster center with the corresponding sphere. Experiments are conducted to validate the proposed method.

\section{Introduction}

Gas turbines, representative equipment of dual rotors, are the key power equipment in aviation, shipping, electric power, petroleum, and so on. Once a gas turbine undergoes a fault or accident, the relevant production and management will suffer a lot. And even worse, those problems will probably lead to fatal disasters $[1,2]$. Therefore, it is very important to ensure the efficient and normal operation of a gas turbine [3].

Vibration monitoring is one of the main methods for mechanical faults monitoring. An early warning of gas turbines' state can be realized through a vibration alarm before a serious fault occurs. To create an alarm for the vibration conditions of certain equipment, the main steps include collecting vibration signals in the main parts of the equipment, computing the vibration overall amplitude, and setting a fixed alarm threshold. The fixed threshold alarm is usually able to ensure the safety and reliable operation for the equipment to some extent. However, this alarm method is mainly suitable for single-rotor equipment under steady operating conditions. This method applies the same alarm threshold under every operating condition and cannot deal with problems that are caused by variable operating conditions. Because the mentioned characteristic of a fixed threshold can easily lead to missing alarms under low operating conditions and false alarms under high operating conditions, this method is not applicable in the warning for the vibration state for a gas turbine under time-varying operating conditions.

Unlike general equipment with one rotor, dual-rotor equipment has two rotors, whose rotational speeds are usually different. A complete description of the operating conditions of rotating machinery should include the speed and the load. The object of this research, however, is dual-rotor equipment including gas turbines and aircraft engines. As for the equipment, the load is the output power or the thrust. Two speeds and load are generated when the rotor is shocked by high temperature and high pressure gas. There is a positive correlation between the speed set and the load. The load does not change 
when the speed set is constant. The load factor is included in the speed factor in this research, so only speed set is analyzed to study the operating conditions for dual-rotor equipment. In engineering applications, the operating conditions of the dual-rotor equipment are usually characterized by rotating speeds. In conclusion, different speeds can be used for characterizing the time-varying operating conditions due to the special research object. Namely, vibration is affected by two different and variable rotational speeds simultaneously, and thus an effective warning for early faults in this type of equipment cannot be realized through the fixed alarm threshold. As previously mentioned, missing and false alarms occur when variable operating conditions are not considered, making traditional alarm methods unable to warn against early faults for this type of equipment effectively. It is necessary to consider the following two aspects of alarm thresholds for dual-rotor equipment: One is to learn alarm threshold values and identify alarm status. And the other is to consider variable operating conditions influenced by two varying rotating speeds.

To solve problems concerning the computation of alarm thresholds and the identification of alarm state, a great number of researches have been carried out for improving the algorithm based on Support Vector Machine (SVM) or Artificial Neural Network (ANN). Empirical Mode Decomposition (EMD) has been applied to obtain the feature set [4]. Then, the ANN has been trained and tested for warning against bearing faults. In recent years, a huge number of scholars have utilized SVM to achieve fault alarms and identify faults. The principle of SVM is that two types of data are separated by finding the optimal hyperplane that has the same distance from itself to both types of data. This means that SVM can warn against faults better than ANN does [5]. SVM, a classifier based on statistical learning theory, was initially proposed to deal with problems when the number of fault samples is not enough [6]. At present, SVM has been used to monitor equipment of various kinds and has been increasingly improved. SVM, whose kernel function is Gaussian, has been utilized to solve classification issues for nonlinear datasets [7], but how to set parameters was still unclear. Immune algorithms that imitate the artificial immune system have been used to optimize parameters of SVM, which plays a significant role in obtaining a classifier with better performance [8]. Several SVMs have been utilized simultaneously to warn against and identify various faults [9]. Then, outputs of each SVM on the basis of the inference of the case database have been obtained. Next, all kinds of the outputs have been compared through the same standard, and the SVM model, suitable for identifying the corresponding fault, has been selected. Those efforts have made great contributions to warning against and identifying faults of bearings. From the above descriptions, it is clear that SVM can achieve higher accuracy and better generalization ability in mechanical fault alarm and recognition [10].

To solve issues caused by variable operating conditions for dual-rotor equipment, a large number of researches focus on how to extract fault features from nonstationary signals in time domain [11-13]. So far, those researches have brought about great benefits. Feature extraction is not concerned in this paper. Nevertheless, there are seldom published researches on early warning methods, which consider varying alarm thresholds caused by operating conditions in dualrotor equipment. The gearbox used in wind turbines is currently mainly studied for the early warning method under time-varying operating conditions. Considering the gearbox under time-varying operating conditions, Ren et al. have obtained influences of speeds and loads on the vibration signal through a large number of experiments, but they have not solved fault warning problems under time-varying operating conditions [14]. Using order tracking and feature extraction in the angle domain, $\mathrm{Gu}$ et al. have obtained the vibration features and load index. Then, they have set up different relevant index models under different operating conditions to recognize gearbox faults, realizing the fault warning under time-varying operating conditions [15]. After extracting the features of the vibration signal at different speeds when the equipment was under normal operating conditions, Lin and Makis have determined the time series models under normal operating conditions. Then, these models have been compared with real-time data to define the status of the equipment. Additionally, load intervals have been divided based on the torque and speed. Then, the gearbox faults were recognized by the Bayesian model [16]. For solving problems of the constantly changeable vibration signal component of the gearbox under time-varying operating conditions, Shao et al. have used an autoregressive model and hypothetical test method to warn against faults in the equipment [17]. Kouadri et al. proposed a method based on the statistical test. This method can define the status of the gearbox by comparing the confidence intervals of the vibration signal between normal and fault experiments. The validity of fault warning for the gearbox under time-varying operating conditions was proved through experimental data [18]. The fault mechanism of dual-rotor equipment is different from that of gearboxes, so the above methods cannot be directly applied to dual-rotor equipment.

This paper proposes an early warning method for dualrotor equipment under time-varying operating conditions using support vector domain description (SVDD) and $K$ means algorithm. To solve problems caused by variable conditions, the range of operating conditions is divided into finite intervals, with each interval considered as a steady operating condition. Alarm thresholds of all intervals are computed one by one. Because the two rotating speeds affect the vibration value simultaneously when the dual-rotor equipment works, SVDD is used to decide the allowable ranges of vibration for the equipment under normal conditions, determining the alarm threshold under each operating condition. Meanwhile, $\mathrm{K}$-means clustering algorithm is used to obtain the cluster center of vibration data whose alarm state is uncertain. The early warning of vibration state can be realized by comparing the cluster center with the alarm threshold under the corresponding operating condition.

The rest of this paper is organized as follows. Section 2 introduces the theoretical backgrounds of this work, including the SVDD, the $K$-means, and the parameter optimization algorithm. Section 3 describes this proposed early warning method. The experimental results are presented to verify this 
proposed method in Section 4. The conclusions are drawn in Section 5. Section 6 presents some discussions about this study.

\section{Basic Theory}

2.1. Support Vector Domain Description. SVM, a statistical learning theory based on machine learning method, can classify data according to structural risk minimization [5]. SVM is mainly utilized for data classification and regression prediction, and this paper only studies the former. SVM is suitable for small sample data, so it is used in this paper to classify data.

Classification problems in engineering practice can be divided into two categories: relatively simple linearly separable problems and linearly inseparable nonlinear problems. SVM initially solves problems when an optimal separating hyperplane is computed in linear separable problems [19]. SVM projects nonlinear separable data onto a high dimension through a nonlinear kernel function to make it a linearly separable problem. Thus, linear distinction of nonlinear data in high dimension will be realized [20].

As a derivative of SVM, one-class SVM is different from binary classification SVM, as it only has one class of data [21]. Currently, there are two types of one-class SVM: one-classSVM and support vector domain description (SVDD). All of them can be used to distinguish abnormal data from normal one [22]. As for SVDD, it is used to discriminate the data by constructing a hypersphere in a high-dimensional space. The center and radius of the hypersphere can be obtained by using the penalty parameter [23]. In this method, spatial features of vibration data under multivariable factors can be shown better. Therefore, SVDD is applied to classify data in this paper.

A training vector $x_{i}\left(x_{i} \in R^{n}, i=1, \ldots, l\right)$ is known and there is no class label; the optimization objective of SVDD is to obtain an optimal hypersphere [24], whose center is $a$ and radius is $R$. It can be expressed as

$$
F\left(R, a, \xi_{i}\right)=R^{2}+C \sum_{i} \xi_{i}
$$

As it is shown in (2), the fixed proportional training data points are included in this sphere.

$$
\left(x_{i}-a\right)^{T}\left(x_{i}-a\right) \leq R^{2}+\xi_{i}, \quad\left(\forall_{i}, \xi_{i} \geq 0\right),
$$

where $\xi_{i}$ represents the relaxation variable. To set $\xi_{i}$ is to prevent interference of individual outliers in the hypersphere. If there is no relaxation variable, the hypersphere will be worse because of few outliers. $C$ is used to adjust the influences of $\xi_{i}$. If $C$ is larger, more outliers will be included. On the contrary, if $C$ is smaller, it is likely that no outliers will be here. Therefore, optimization of $C$ is of great significance. This problem will be explained in Section 2.3.

The hypersphere can solve nonlinear problems. For this purpose, data points must be projected onto highdimensional space for finding the optimal hyperplane, that is, a kernel function $k\left(x, x^{\prime}\right)$, which can satisfy the following equation:

$$
k\left(x, x^{\prime}\right)=\varphi(x) \varphi\left(x^{\prime}\right),
$$

where $x$ and $x^{\prime}$ denote coordinates of the data and $\varphi(\cdot)$ represents the function which can project the coordinate onto high-dimensional space. Thus, the optimization problem is shown as follows:

$$
\min R^{2}+C \sum_{i=1}^{N} \xi_{i}
$$

which is subjected to

$$
\begin{aligned}
{\left[\varphi\left(\varphi_{i}\right)-a\right]\left[\varphi\left(\varphi_{i}\right)-a\right]^{T} \leq R^{2}+\xi_{i}, } & \\
& \left(\xi_{i} \geq 0, i=1, \ldots, N\right) .
\end{aligned}
$$

$N$ in (4)-(5) denotes the number of training samples. The dual form of (5) is shown as

$$
\min \sum_{i, j=1}^{N} \alpha_{i} \alpha_{j} k\left(x_{i}, x_{j}\right)-\sum_{i=1}^{N} \alpha_{i} k\left(x_{i}, x_{i}\right) .
$$

The constraint of (6) is

$$
\sum_{i=1}^{N} \alpha_{i}=1, \quad\left(0 \leq \alpha_{i} \leq C, i=1, \ldots, N\right),
$$

where $\alpha_{i}$ denotes the coefficient of Lagrange function. In solving the above optimization problem, most $\alpha_{i}$ is zero. If $\alpha_{i}$ is nonzero, it is a support vector, which decides the shape and size of the hypersphere. Based on all the support vectors, $a$, the center of the sphere, is shown as follows:

$$
a=\sum_{i \in I_{S V}} \alpha_{i} \varphi\left(x_{i}\right),
$$

where $I_{\mathrm{SV}}$ represents the set of support vectors. As for support vector with $0 \leq \alpha_{i} \leq C, x_{i}$ satisfies

$$
R^{2}-\left[k\left(x_{i}, x_{i}\right)-2 \sum_{j \in I_{\mathrm{SV}}} \alpha_{j} k\left(x_{j}, x_{i}\right)+C^{2}\right]=0 .
$$

The radius $R$ of the sphere can be obtained from the above equation.

As mentioned earlier, the kernel function can be divided into two types: linear and nonlinear. Nonlinear kernel functions include polynomial, Gaussian, sigmoid, and selfdefined types. The Gaussian kernel function, one of the most commonly used kernel functions, can be used easily and nearly without problems of numerical solutions [25]. Thus, the Gaussian kernel function is used as a mapping function in high dimension in this paper, as it is shown in

$$
e^{-\gamma|u-v|^{2}}
$$


where $u$ denotes the sample point, $v$ represents the center decided by the sample points, and $\gamma$ denotes the parameter which decides the change rate of the kernel function. When $\gamma$ becomes larger, the corresponding parting surface will be more complex. On the contrary, when $\gamma$ is less, the relevant parting surface will become smoother. Therefore, choosing the value of $\gamma$ is also very important. This problem will be explained in Section 2.3.

2.2. K-Means Clustering Algorithm. The clustering algorithm is to determine the distribution of data through a statistical method. The distribution can be regarded as a certain kind of geometry, and the cluster center is the barycenter of the geometry $[26,27]$. Actually, it is difficult to find this center in many data. Meanwhile, the dataset must be divided into several different classes. $K$-means clustering algorithm has been initially introduced and has been applied widely into various research fields [28]. In this paper, the cluster center of test data can be found in three-dimensional spaces via the $K$-means clustering algorithm. When compared with the distribution of the data points, the cluster center of data is a more stable feature and can characterize the state of equipment better [29]. This is because misjudgment due to individual points is avoided by the cluster center.

$X=\left\{x_{1}, x_{2}, \ldots, x_{i}, \ldots, x_{l}\right\}$ is a $d$-dimensional dataset including $l$ samples, where $x_{i} \in R^{d} . K$ in the $K$-means algorithm represents the notion that $X$ is divided into $K$ subclasses. Each subclass is expressed as $c_{i}(i=1,2, \ldots, K)$, and every $c_{i}$ has its own cluster center $u_{i}$. Firstly, select $K$ elements randomly to be the initial cluster centers of $K$ subclasses. Then, compute the distance from each data point to every subclass center. The initial classification can be realized according to the criterion of the shortest distance. Next, calculate the average Euclidean distance of each subclass, update the cluster center on account of this average, and renew the classification according to the principle of the shortest distance. Equations (11) and (12) are, respectively, the quadratic sum of distance from the data point in each subclass to the corresponding class center $u_{i}$ and the quadratic sum of the total distance of all classes [30].

$$
\begin{aligned}
J\left(c_{k}\right) & =\sum_{x_{i} \in c_{k}}\left\|x_{i}-u_{k}\right\|^{2} \\
J(C) & =\sum_{k=1}^{K} J\left(c_{k}\right)=\sum_{k=1}^{K} \sum_{x_{i} \in \epsilon_{k}}\left\|x_{i}-u_{k}\right\|^{2} \\
& =\sum_{k=1}^{K} \sum_{i=1}^{l} d_{k i}\left\|x_{i}-u_{k}\right\|^{2},
\end{aligned}
$$

where

$$
d_{k i}= \begin{cases}1, & x_{i} \in c_{i} \\ 0, & x_{i} \notin c .\end{cases}
$$

$\mathrm{K}$-means clustering algorithm aims at minimizing the quadratic sum of distance of all classes. Update the cluster centers and classifications constantly according to the above

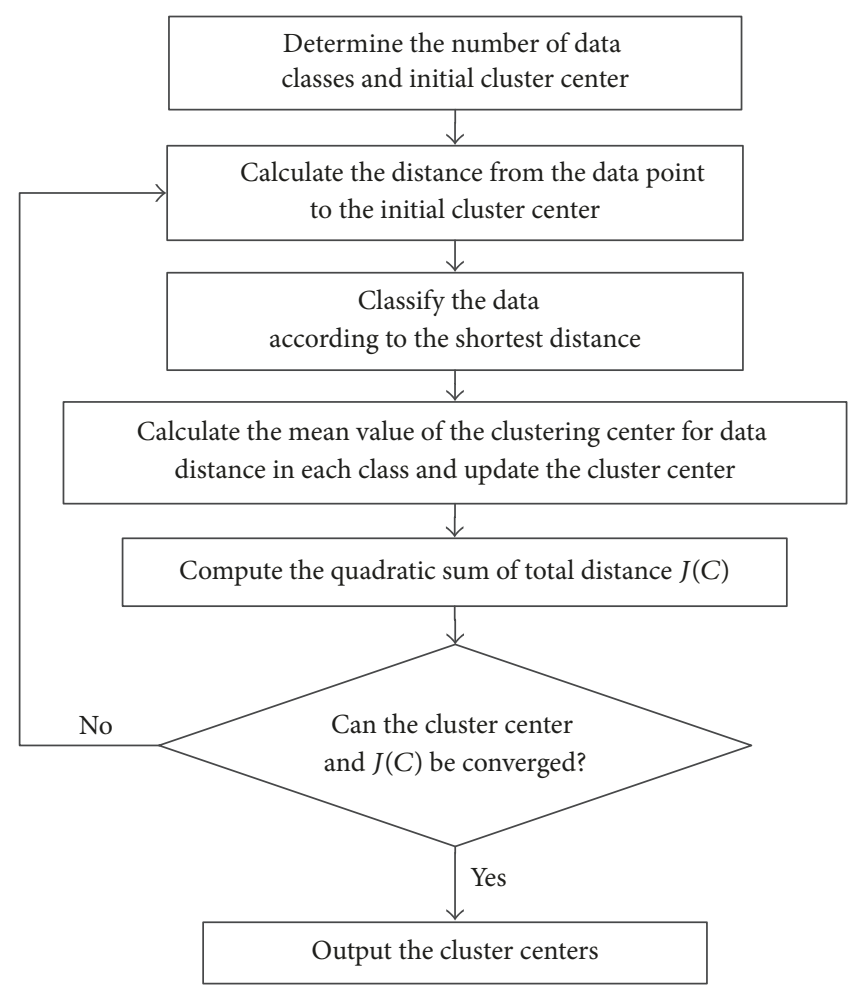

FIGURE 1: Flow chart of the $K$-means clustering algorithm.

steps when the quadratic sum is convergent. The flow chart of the $K$-means clustering algorithm is shown in Figure 1.

2.3. Parameter Optimization Algorithm. As per the description in Section 2.1, the penalty factor $C$ adjusts the confidence interval when SVDD defines the data subspace, and it is also the balance between the misclassification ratio and the algorithm complexity [31]. When $C$ is smaller, the algorithm is less complex and the empirical risk is greater. With the increase of $C$, the complexity is increased and the value of the experience risk is reduced. When $C$ is larger, the classification results of datasets which have large samples are unsatisfactory. Therefore, the value of $C$ should be appropriate. Each data should have at least one reasonable value of $C$ to make the best generalization performance of SVDD.

According to Section 2.1, linearly inseparable datasets can be converted to linearly separable datasets through a kernel function [31]. The Gaussian kernel function selected in this paper has a few parameters and a high classification accuracy. Parameter $\gamma$ in the kernel function affects the nonlinear transformation function, and its change will affect the distribution dimension of the sample data in the space. If $\gamma$ is large, the value of the kernel function will approach 0 , which will lead to overlearning. In other words, the classifier can only correctly classify the training samples and fails to classify the unknown testing samples. On the contrary, less learning will occur, and all testing samples will be classified into one class, which leads to a wrong classification.

From the above, the penalty factors $C$ and $\gamma$ play a significant role in the performance of SVDD. Therefore, the 
property of SVDD will be improved to a great extent if a more appropriate parameter set $(C, \gamma)$ is selected.

The question of which is the best parameter optimization method for SVDD still has no answer in academic circles. The commonly used methods for optimizing SVDD's parameters are Grid Search, Genetic Algorithm, and Particle Swarm Optimization [32-34].

Each of the three optimization algorithms has its advantages. If the search interval is large enough and the search step is small enough, the Grid Search can find the global optimal solution. However, it will take a long time to traverse all the parameter groups in the grid. The Genetic Algorithm and Particle Swarm Optimization are heuristic algorithms. They can find the global optimal solution without traversing all the parameter groups in the interval. However, the operation of the two algorithms is often complex, and it is easy for them to fall into the local optimum rather than the global optimal solution. When the number of samples contained in the dataset is small, it is better to use Grid Search to optimize the parameters of SVDD. On the contrary, the Genetic Algorithm and Particle Swarm Optimization are better. The computation time required for GA is longer than that of PSO, but the classification accuracy of GA is higher.

Therefore, different algorithms may be used for obtaining the best results in different applications or operating conditions. In order to obtain the best results as much as possible, three algorithms are selected for optimization and the most effective one is selected under each condition. Therefore, it is necessary to determine them through three optimization algorithms.

2.3.1. Grid Search. The processes of optimization algorithm of Grid Search are as follows. Firstly, set ranges of parameters $C$ and $\gamma$; these two parameters, respectively, correspond to the horizontal and vertical axes of a planar coordinate system. Then, set a certain step length for the two parameters and obtain a value and draw a line on the coordinate diagram according to the parameters. Finally, there will be a grid, which can determine the final optimal parameters through the obtained parameters in the grid point [35]. This method, however, is used not only to calculate results but also to make cross-validation. The procedures of Grid Search are shown in Figure 2.

2.3.2. Genetic Algorithm. The genetic parameters optimization algorithm can be realized according to Darwin's theory of evolution, survival of the fittest. Similar to human gene evolution, the Genetic Algorithm can achieve parameter changes according to imitating competition between superior and degradation genes, selection of superior genes, combination of genes, and genovariation. Additionally, it computes the fitness value of population parameters through the fitness function. Higher fitness value shows that the parameter is better [36]. Firstly, the Genetic Algorithm generates a parameter population randomly. Then, relatively optimal parameters can be selected via the fitness function and the corresponding parameter population will be obtained. Parameters can be updated using the above steps and the iteration terminating condition can be set according to the actual requirements.

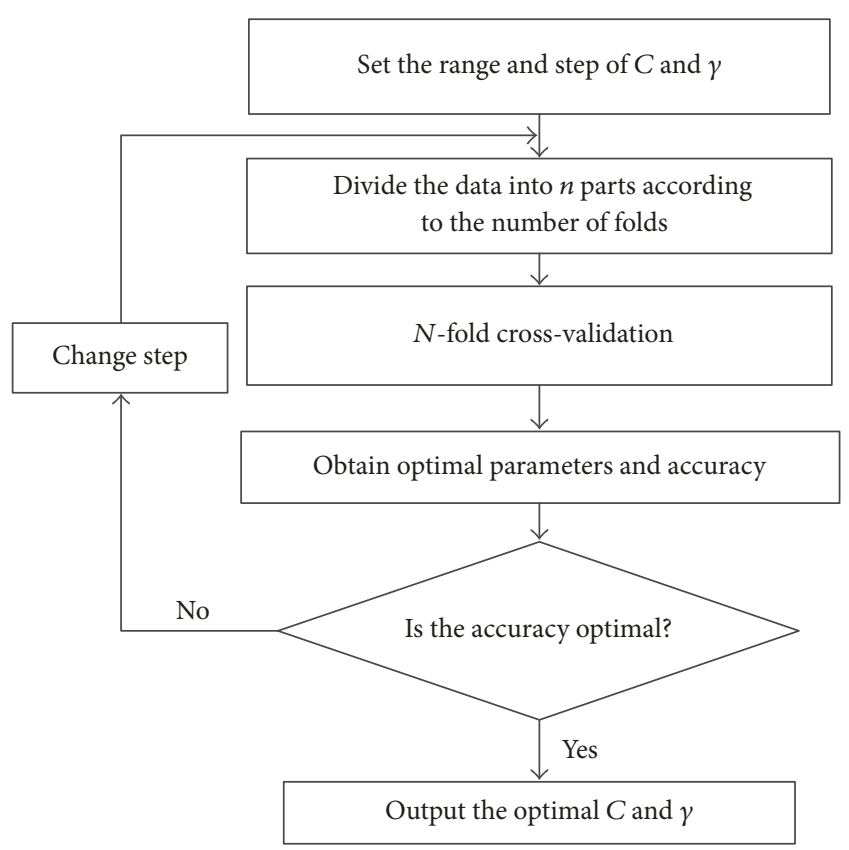

FIGURE 2: Flow chart of Grid Search.

Once the iteration is terminated, optimal parameters that satisfy certain conditions will be obtained [37].

The flow chart of a commonly used Genetic Algorithm is shown in Figure 3.

2.3.3. Particle Swarm Optimization. Particle Swarm Optimization (PSO) mainly simulates migration and aggregation behaviors of birds foraging. Similar to the Genetic Algorithm, PSO seeks the optimal particle as the last parameter in the constant iteration [38]. Firstly, a group of random particles are selected as the initial solution. During the iteration, the solution can be updated when particles track two extremums. One is the extremum of the particles themselves and can be called the individual extremum. The other is the one that has been found from the whole population. Advancing direction and speed of those particles can be updated by constantly computing optimal values of particles and the population. Those steps make the particle swarm continuously move towards the direction of the optimal solution. Thus, the optimal solution of parameters can be found ultimately [39]. The flow chart of PSO is shown in Figure 4.

2.3.4. Cross-Validation Accuracy. The parameter optimization algorithm which is selected in each operating condition is chosen from the above three algorithms according to the maximum cross-validation accuracy. These algorithms all divide the dataset into $n$ subsets and regard the $n-1$ subsets as training ones. The left one is used as a prediction set to judge the above training state. The $n$ subsets all need prediction sets for their relevant accuracies, and the accuracy of the algorithm can be obtained by computing the average of these 


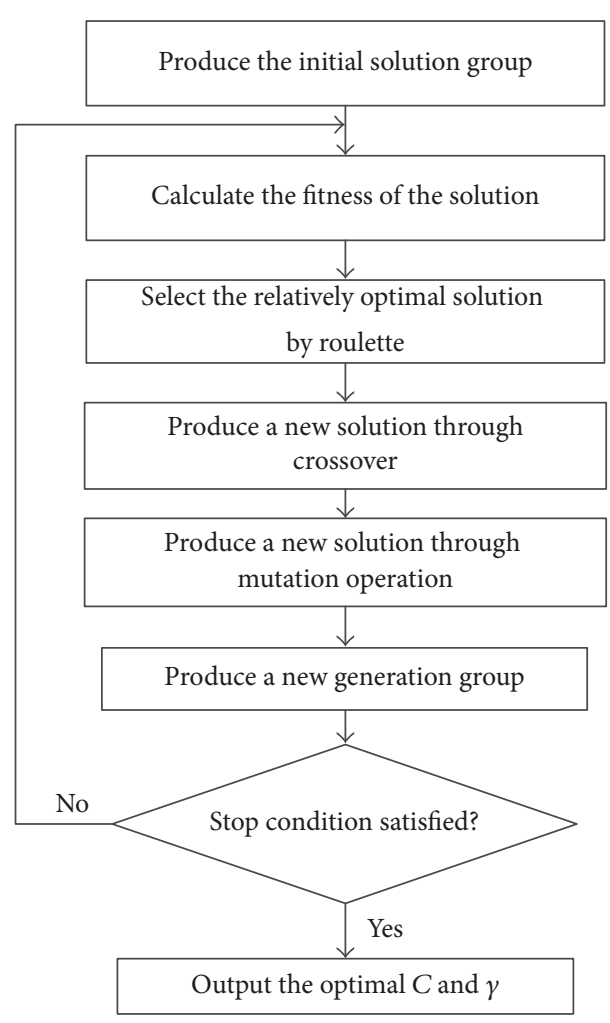

FIgUre 3: Flow chart of the Genetic Algorithm.

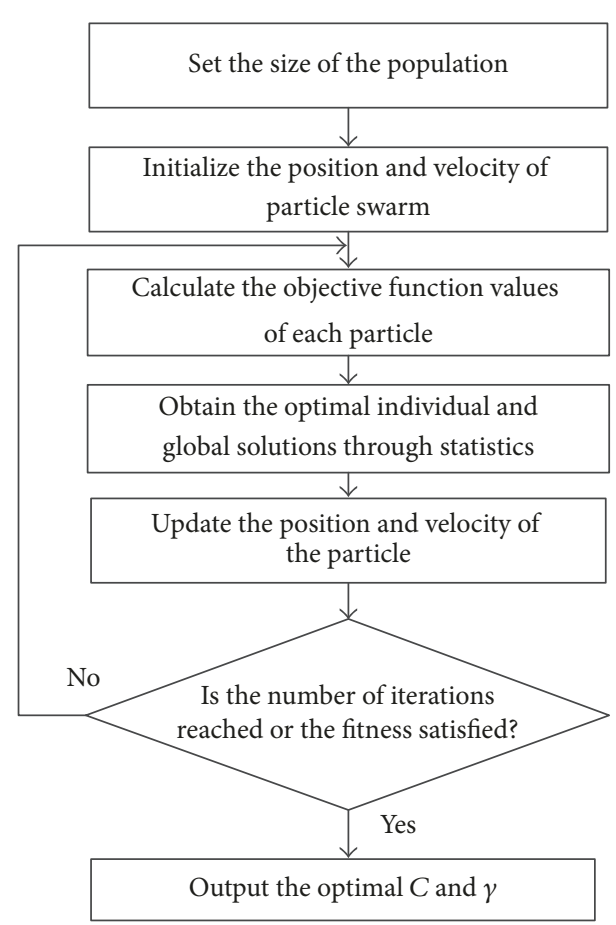

FIgURE 4: Flow chart of the PSO algorithm.

above accuracies [40]. The cross-validation accuracy $e_{n}$ is shown in

$$
e_{n}=\frac{1}{n} \sum_{i=1}^{n}\left[\frac{\operatorname{num}\left(y_{i}=\widehat{y}_{i}\right)}{m}\right] \text {, }
$$

where $m$ denotes the number of data in each subset; $y_{i}$ and $\hat{y}_{i}$ represent the actual value and predicated value of the data element, respectively; num $(\cdot)$ denotes the number of data elements in which the condition · is satisfied.

\section{The Proposed Method for Early Warning}

This paper studies an early warning method for dual-rotor equipment under time-varying operating conditions. The method is based on SVDD and $K$-means algorithm, as represented graphically in Figure 5. The main procedures are expressed as follows.

(1) Interval Partition of Baseline Vibration. When a piece of equipment works normally, vibration feature values such as peak or RMS of acceleration, RMS or peak of velocity, and peak to peak of displacement are divided into several intervals according to the dual-rotor speeds. Under an interval of an operating speed, $l$ signal sequences can be described as $S_{i}=\left[N 1_{i}, N 2_{i}, A s_{i}\right], i=1,2, \ldots, l$, where $l$ is the number of datasets within this operating condition (running speed) interval. The three elements of $S_{i}$ are, respectively, rotating speed 1 , rotating speed 2 , and vibration feature.

(2) Optimization of Parameters $C$ and $\gamma$ in SVDD. $l$ signal sequences $S_{i}$ are inputs of Grid Search, Genetic Algorithm, and Particle Swarm Optimization within an operating condition interval. After the parameters are optimized, their results and accuracies can be shown as $\left[C_{1}, \gamma_{1}, e_{1}\right],\left[C_{2}, \gamma_{2}, e_{2}\right]$, and $\left[C_{3}, \gamma_{3}, e_{3}\right]$. Then, select $C$ and $\gamma$ with the highest accuracy rate among $e_{1}, e_{2}$, and $e_{3}$. They can be regarded as the optimum parameters. The parameters in every interval of an operating condition should be optimized as the above steps and the optimal solution of $C$ and $\gamma$ under each operating condition will be obtained.

(3) Calculation of Alarm Threshold Parameters $O_{i a}$ and $R_{i a}$. Regard $l$ signal sequences $S_{i}$, under an operating condition range, as the input of SVDD. Insert the optimal solutions $C_{o}$ of $C$ and $\gamma_{o}$ of $\gamma$ into (8), (9), and (10). Then, the center $O_{i a}$ and the radius $R_{i a}$ of the optimal sphere will be known. $O_{i a}$ and $R_{i a}$ can show the normal variation range of vibration data for equipment under an operating condition range; this normal range can be defined as the alarm threshold value under this operating condition range. Apply the above calculation to each range of the operating condition to obtain all alarm threshold values.

(4) Determination of Cluster Center of the Test Data Whose Alarm State Is Unknown. The test dataset whose alarm state is unknown is taken as the input of $K$-means algorithm. Cluster center $O_{N}=\left(N 1_{N}, N 2_{N}, A S_{N}\right)$ can be obtained according to (12). This cluster center can denote the operating state of the equipment steadily and reduce false alarms caused by few outliers as much as possible.

(5) Judgment of the Alarm State. When the distance $r_{N}$ from $O_{N}$, cluster center of the test data, to $O_{i a}$, the center of the optimal sphere in the corresponding operating condition 


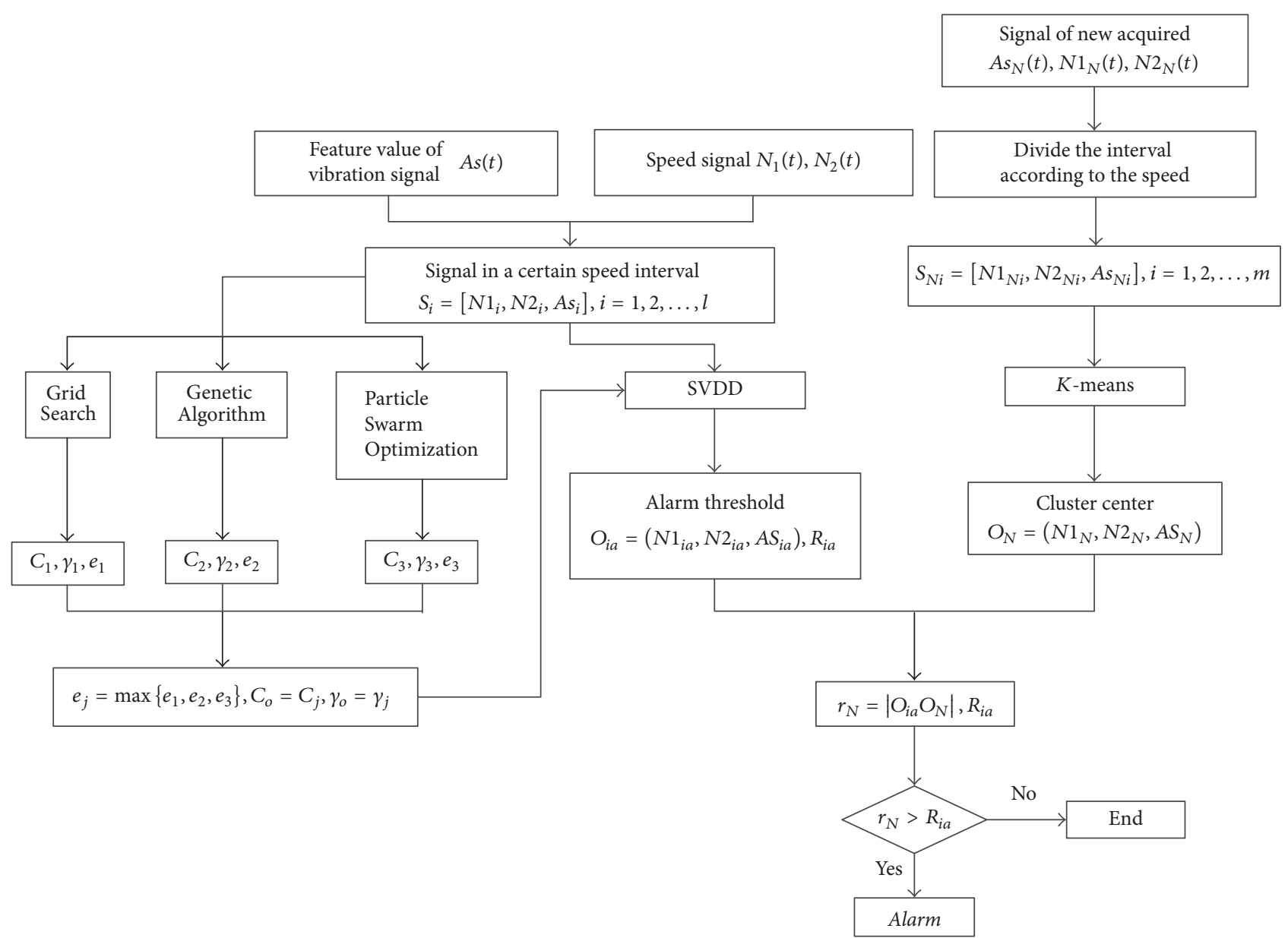

FIgURE 5: Schematic of the proposed method.

range, satisfies the following condition that $r_{N} \leq R_{i a}$, the equipment can be considered to work normally. Oppositely, if $r_{N}>R_{i a}$, it means that the equipment is on alarm.

\section{Experiments}

A dual-rotor test rig has been set up to simulate the operating environment of the real dual-rotor equipment. To carry out the experimental study, two datasets of vibration in bearing housing and rotating speeds of dual rotors have been collected, respectively, when there were no faults and defects in $1 \#$ intershaft bearing.

4.1. Experiments Setup. The dual-rotor test rig, applied in the experiment, is shown in Figure 6.

In the experiment, intershaft bearings under normal and fault conditions are installed. The experiment can be divided into two groups: (1) the test rig under normal conditions and (2) faults in the outer ring of the intershaft bearing $(1 \mathrm{~mm}$ deep and $1 \mathrm{~mm}$ wide groove (simulation of initial fault) across the axis on the inner surface of the outer ring).

The vibration data is collected by LMS SCADAS [41]. The vibration sensor is BK4519 accelerometer, and the key

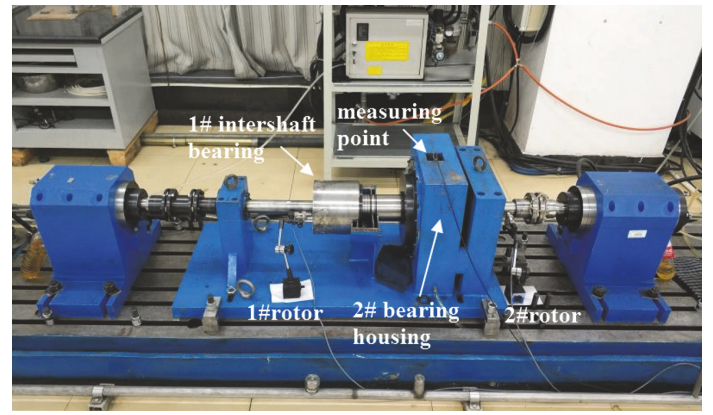

Figure 6: Test rig for dual-rotor faults simulation.

phase sensor is a proximity switch. The accelerometer has been mounted vertically on the 2\# bearing housing. Speeds of $1 \#$ rotor and $2 \#$ rotor can be controlled through two motorized spindles. Vibration and rotating speed signals can be collected when dual-rotor speeds are variable.

The selected data to be processed in the following part can be divided into two groups: (1) data under normal conditions (the rotating speed $N_{1}$ of $1 \#$ rotor slightly fluctuates at $300 \mathrm{rpm}, 900 \mathrm{rpm}$, and $1500 \mathrm{rpm}$; the rotating speed $\mathrm{N}_{2}$ of 2\# 

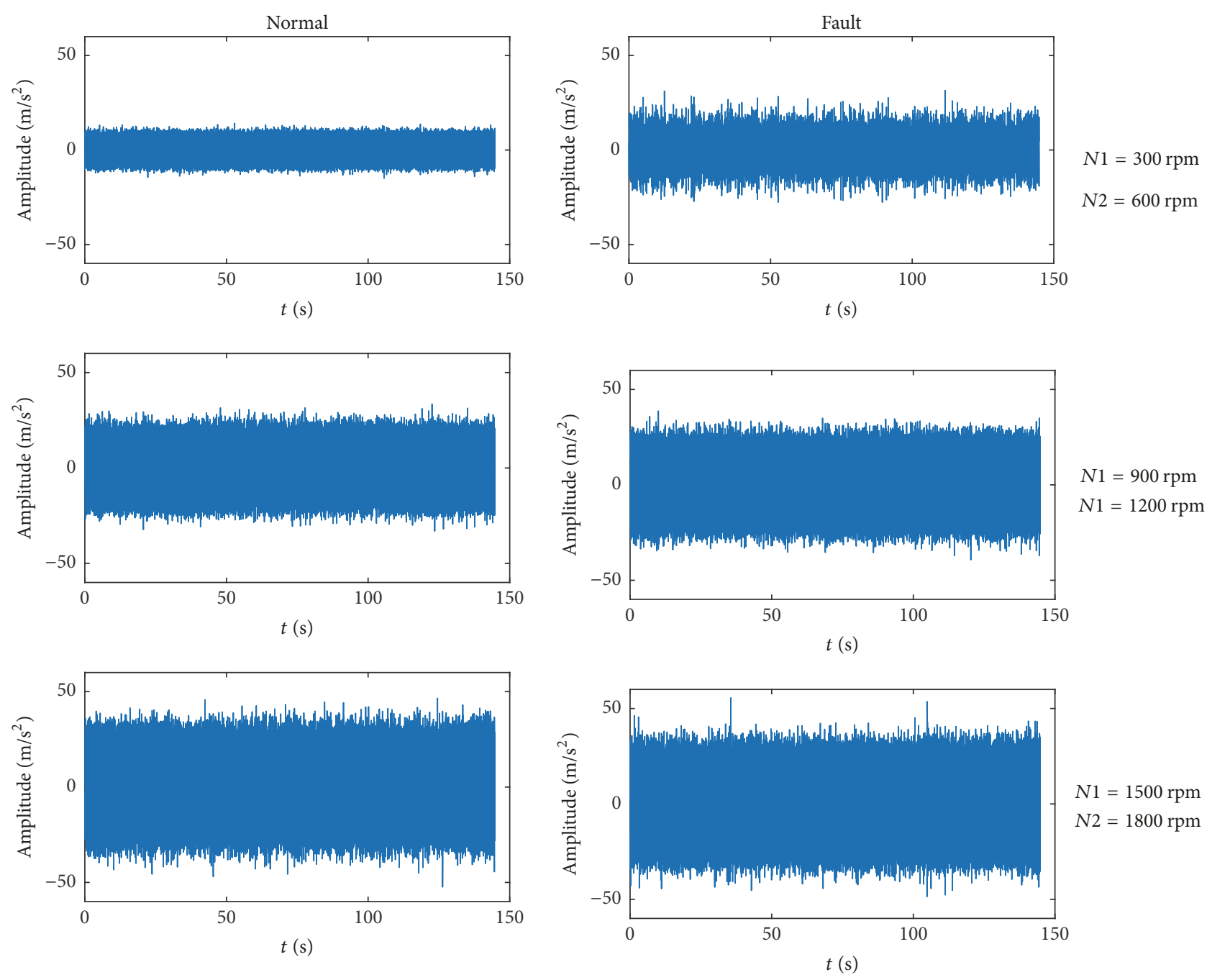

FIGURE 7: Waveforms of normal and fault vibration signals.

rotor slightly fluctuates at $600 \mathrm{rpm}, 1200 \mathrm{rpm}$, and $1800 \mathrm{rpm}$, resp.; the sampling rate is $25.6 \mathrm{kHz}$ ) and (2) fault data of the outer ring in the intershaft bearing (the operating condition and sampling rate are the same with the above data). The waveforms of normal and fault vibration signals at different speeds are shown in Figure 7.

Because RMS can steadily represent the operating state of equipment, this paper selects RMS of acceleration as the vibration feature value. It can be calculated by (14).

$$
X_{\mathrm{rms}}=\sqrt{\frac{\sum_{i=1}^{N} X_{i}^{2}}{N}} .
$$

4.2. Analysis through Fixed Threshold Alarm. The fixed threshold alarm means that one threshold is adopted to warn for the equipment under every operating condition without considering influences of time-varying conditions. In this section, a fixed alarm threshold has been set according to vibration data of the dual-rotor test rig under normal condition. Vibration acceleration can be selected when the test rig works at normal conditions: 1 \# rotor speed is $900 \mathrm{r} / \mathrm{min}$ and
TABLE 1: Fixed alarm threshold for the test rig.

\begin{tabular}{lccc}
\hline & 1\# rotor & 2\# rotor \\
\hline Speed $(\mathrm{r} / \mathrm{min})$ & 900 & & 1200 \\
Max. RMS $\left(\mathrm{m} / \mathrm{s}^{2}\right)$ & & 6.32 & \\
Alarm threshold $\left(\mathrm{m} / \mathrm{s}^{2}\right)$ & & 7.90 & \\
\hline
\end{tabular}

2\# rotor speed is $1200 \mathrm{r} / \mathrm{min}$. Then, the RMS can be computed. The alarm threshold is 1.25 times the maximum RMS [42], as is shown in Table 1.

Fault data of intershaft bearings are analyzed when the rotating speed of $1 \#$ rotor is $300 \mathrm{r} / \mathrm{min}$ and that of $2 \#$ rotor is $600 \mathrm{r} / \mathrm{min}$. The RMS of vibration acceleration is about $3 \mathrm{~m} / \mathrm{s}^{2}$, which is the fault data at a low speed, as is shown in Figure 7. The figure shows that fault data, under this operating condition, is lower than the fixed alarm threshold, $7.9 \mathrm{~m} / \mathrm{s}^{2}$. Thus, the above fixed threshold alarm cannot accurately warn against initial faults in low operating conditions.

The data under the normal condition can also be analyzed when the rotating speed of $1 \#$ rotor is $1500 \mathrm{r} / \mathrm{min}$ and that 


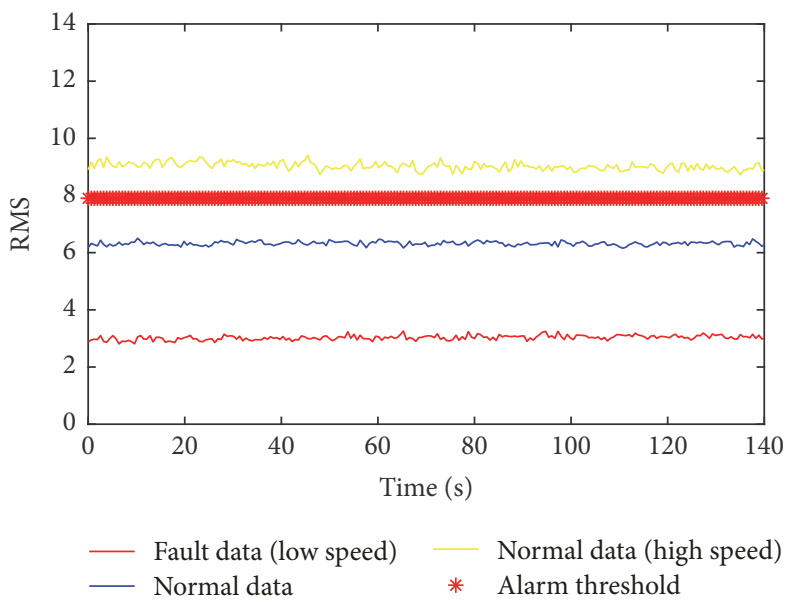

Figure 8: Analysis results of the fixed threshold alarm.

TABLE 2: Interval division of experimental data.

\begin{tabular}{lccc}
\hline Operating condition & 1 & 2 & 3 \\
Speed of 1\# rotor $(\mathrm{rpm})$ & 300 & 900 & 1500 \\
Speed of 2\# rotor $(\mathrm{rpm})$ & 600 & 1200 & 1800 \\
\hline
\end{tabular}

of 2 \# rotor is $1800 \mathrm{r} / \mathrm{min}$. The RMS of acceleration is about $9 \mathrm{~m} / \mathrm{s}^{2}$. It is shown as normal data at a high speed in Figure 8. From the figure, it is obvious that the data under this condition is higher than the fixed alarm threshold, $7.9 \mathrm{~m} / \mathrm{s}^{2}$. Thus, a false alarm is produced through the alarm method based on the fixed threshold under high operating conditions.

From the above analysis, influences from two rotating speeds of the dual-rotor equipment on vibration are ignored by the fixed threshold alarm method. Hence, this method is commonly applied to equipment with a constant speed, but it is inadaptable to warn against faults for dual-rotor ones. A higher value of the fixed threshold means that it is easier for equipment with faults to miss fault warning under low operating conditions; namely, the missing alarm rate will be higher. Similarly, a lower value of the fixed threshold means that it is easier for equipment without faults to warn under high operating conditions; namely, the false alarm rate will be higher. Utilizing a fixed threshold to warn for the equipment will make it difficult to keep the balance between missing alarm rate and false alarm rate.

4.3. Analysis by the Proposed Method. The algorithm proposed in this paper is used to analyze the data of the test rig. Processes are as follows.

4.3.1. Interval Division of Baseline Vibration Features. The data, under normal and fault conditions, are divided into three groups according to the dual-rotor rotating speeds, as shown in Table 2.

4.3.2. Parameter Optimization. The experimental data without faults, under the above three operating conditions, have been analyzed through Grid Search, Genetic Algorithm, and
TABLE 3: Parameters setting and optimization results of Grid Search.

\begin{tabular}{lcc}
\hline Parameters & $C$ & $\gamma$ \\
Range of values & {$\left[2^{-8}, 2^{8}\right]$} & {$\left[2^{-8}, 2^{8}\right]$} \\
Step & 1 & 1 \\
Optimization result & 0.5 & 0.25 \\
Fold of cross-validation & \multicolumn{2}{c}{10} \\
Cross-validation accuracy & \multicolumn{3}{c}{$98.3516 \%$} \\
\hline
\end{tabular}

Particle Swarm Optimization. This is to obtain parameters $C$ and $\gamma$ under each operating condition for SVDD algorithm. Take operating condition 1 as an example; the processes of parameter optimization are shown as follows.

(1) Grid Search. In order to achieve optimum parameters $C$ and $\gamma$, Grid Search is utilized to analyze three-dimensional data under operating condition 1 . Those data include dualrotor speeds and RMS of the acceleration. A contour map and the $3 \mathrm{D}$ chart of SVC selection results can be obtained as soon as the parameters are optimized, as is shown in Figure 9. The settings of main parameters and optimization results are shown in Table 3.

(2) Genetic Algorithm. The Genetic Algorithm is used to analyze three-dimensional data including dual-rotor speeds and RMS of the acceleration under operating condition 1. A fitness curve can be obtained, as is shown in Figure 10. Main parameters' setting and optimization results are shown in Table 4.

(3) Particle Swarm Optimization. Particle Swarm Optimization is applied to analyzing three-dimensional data including dual-rotor speeds and RMS of the acceleration under operating condition 1 . Then, a fitness curve can be obtained, as is shown in Figure 11. The settings of main parameters and optimization results are shown in Table 5.

A cross-validation accuracy of $98.8889 \%$, obtained through Particle Swarm Optimization, is the highest value among all the accuracies of all three optimization algorithms 


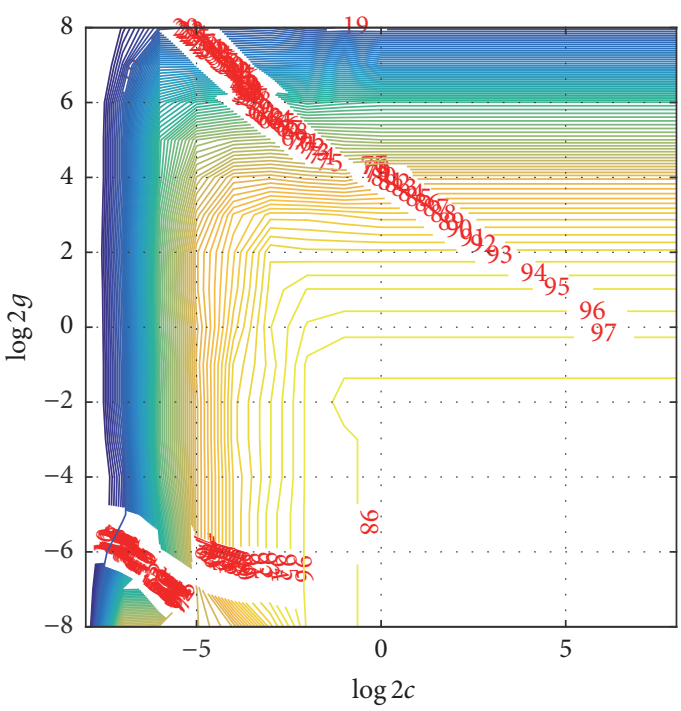

(a)

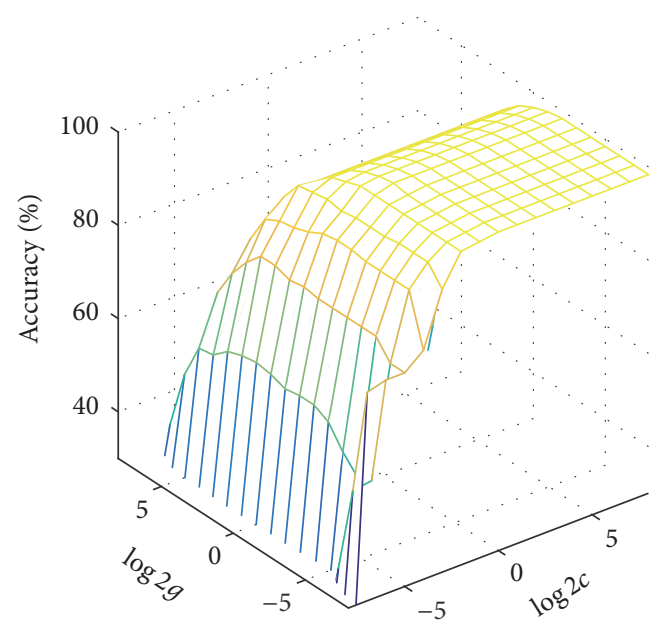

(b)

FIGURE 9: Results of the Grid Search algorithm: (a) contour chart; (b) 3D view.

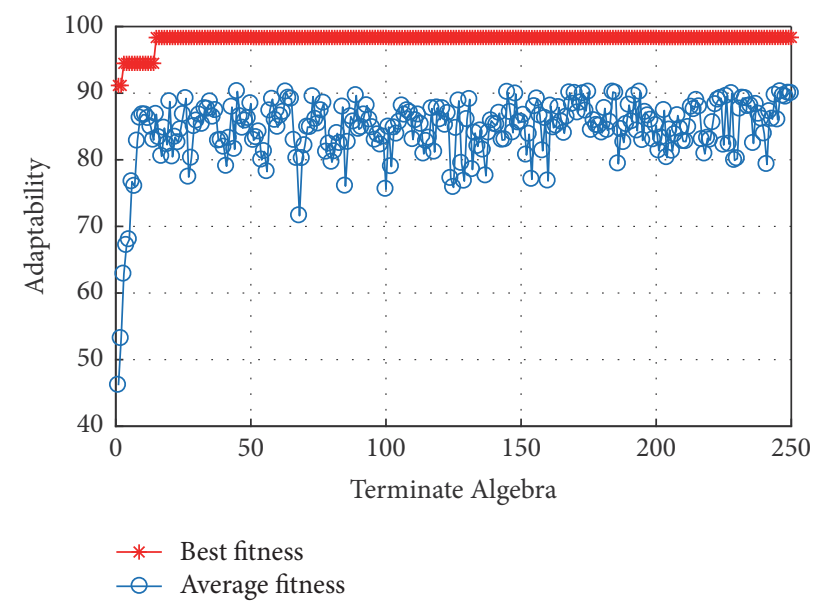

Figure 10: Fitness curve of the Genetic Algorithm.

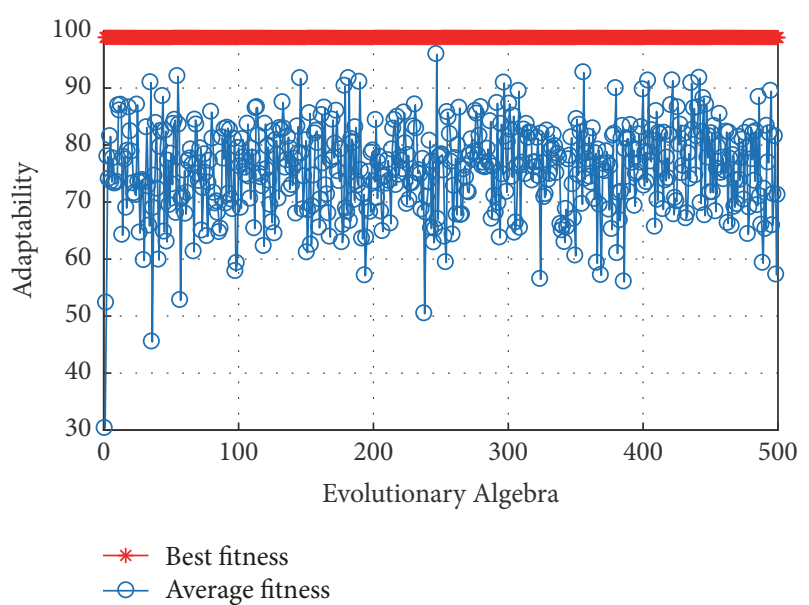

FIgURE 11: Fitness curve of Particle Swarm Optimization.
TABLE 4: Parameters setting and optimization results of the Genetic Algorithm.

\begin{tabular}{lccc}
\hline Parameters & $C$ & & $\gamma$ \\
Range of values & {$[0,256]$} & & {$[0,256]$} \\
Maximum population & & 20 & \\
Maximum generation & & 500 & \\
Fold of cross-validation & & 10 & \\
Optimization results & 0.74438 & \multicolumn{2}{c}{0.17261} \\
Cross-validation accuracy & \multicolumn{3}{c}{$98.3425 \%$} \\
\hline
\end{tabular}

TABLE 5: Parameters setting and optimization results of PSO.

\begin{tabular}{lccc}
\hline Parameters & $C$ & \multicolumn{2}{c}{$g$} \\
Range of values & {$[0.1,100]$} & \multicolumn{2}{c}{$[0.01,1000]$} \\
Maximum evolution & & 500 & \\
Maximum population & & 20 & \\
Local search speed & & 1.5 & \\
Global search speed & & 1.7 & \\
Fold of cross-validation & & 7 & 0.01 \\
Optimization results & 0.52958 & \multicolumn{3}{c}{} \\
Cross-validation accuracy & & \multicolumn{3}{c}{$98.8889 \%$} \\
\hline
\end{tabular}

that have been used to process experimental data without faults under operating condition 1. Thus, under this operating condition, optimization results of Particle Swarm Optimization are regarded as the values of $C$ and $\gamma$; namely, $C_{1}=0.52985$ and $\gamma_{1}=0.01$.

Under each operating condition, those three optimization algorithms can be utilized to determine $C$ and $\gamma$. Take parameters obtained through an algorithm whose crossvalidation accuracy is the highest among all the accuracies as the trained parameters of SVDD. The final results are shown with boldface letters in Table 6 . 
TABLE 6: Results of parameter optimization.

\begin{tabular}{|c|c|c|c|c|}
\hline \multicolumn{2}{|c|}{ Operating condition } & 1 & 2 & 3 \\
\hline \multirow{3}{*}{ Grid Search } & Accuracy (\%) & 98.3516 & 96.6667 & 98.3333 \\
\hline & C & 0.5 & 0.5 & 0.5 \\
\hline & $\gamma$ & 0.25 & 0.0039063 & 0.0039063 \\
\hline \multirow{3}{*}{ Genetic Algorithm } & Accuracy (\%) & 98.3425 & 98.3471 & 98.3471 \\
\hline & C & 0.74438 & 1.332 & 2.3611 \\
\hline & $\gamma$ & 0.17261 & 0.087738 & 0.099182 \\
\hline \multirow{3}{*}{ Particle Swarm Optimization } & Accuracy (\%) & 98.8889 & 96.6667 & 98.3333 \\
\hline & C & 0.52985 & 0.912166 & 1.0021 \\
\hline & $\gamma$ & 0.01 & 0.01 & 0.01 \\
\hline
\end{tabular}

TABLE 7: Alarm threshold of the dual-rotor test rig.

\begin{tabular}{lccc}
\hline Operating condition & 1 & 2 & 3 \\
Center of sphere & {$[300,600,2.79648]$} & {$[897,1199,6.30922]$} & {$[1502,1803,9.0504]$} \\
Radius of sphere & 0.2023 & 0.6035 & 0.6362 \\
\hline
\end{tabular}

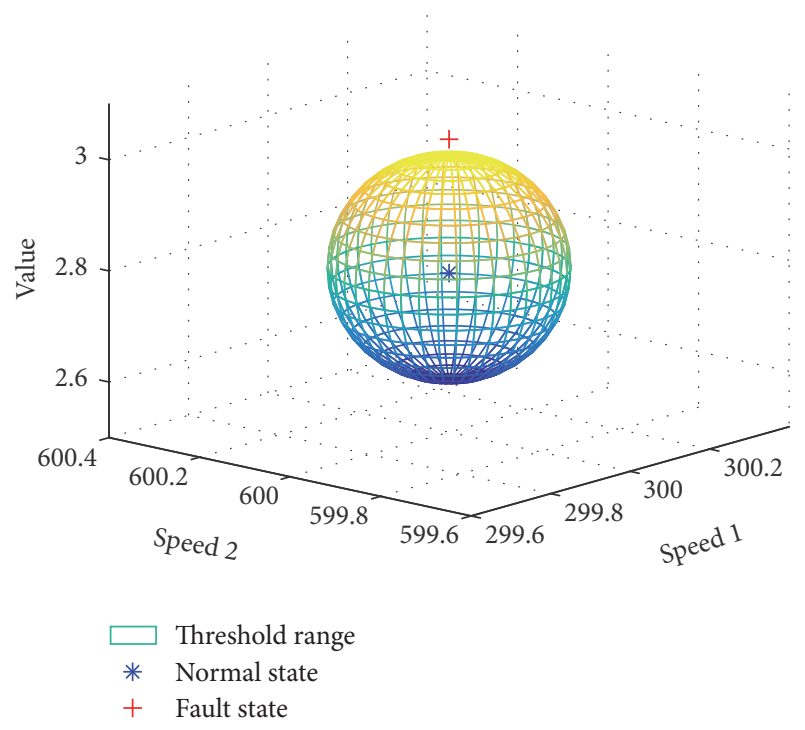

Figure 12: Alarm system simulation.

4.3.3. Determination of the Alarm Threshold. Optimization results of $C$ and $\gamma$ in Section 4.3.2 are adopted by the SVDD algorithm to process experimental data without faults under three operating conditions in sequence. Hence, alarm threshold values under each operating condition are obtained. The alarm threshold can be shown as a sphere, whose controlling parameters are the coordinate of center and the radius. Take operating condition 1 as an example; the sphere representing the alarm threshold is shown in Figure 12. When the data is in the inner part or on the surface of the sphere, as the blue point shown in the figure, the equipment works at a normal condition. However, when data is on the outside of the sphere, as the red point shown in the figure, the equipment is in a state of alarm.
Alarm threshold values of the test rig under all the operating conditions can be obtained through the SVDD algorithm, as is shown in Table 7.

4.3.4. Judgment of the Alarm State. The cluster center of experimental data with and without faults under the above three operating conditions can be computed through the $K$ means algorithm. Then, distances from all the cluster centers to the corresponding centers of the sphere can be calculated, as is shown in Table 8.

Compare the distance from the cluster center in Table 8 to the center of the sphere with the radius of the sphere in Table 7. If the distance is longer than the radius of the sphere, the equipment is in a state of alarm. On the contrary, if the distance is shorter than the radius of the sphere, the equipment works at a normal condition.

The distances, obtained on the basis of experimental data without faults under each operating condition, are compared with the corresponding radius of the sphere. As is shown in Figure 13, yellow cylinders representing the distances are lower than the red cylinders which represent alarm threshold values; therefore, this equipment works normally under all operating conditions. This analysis result corresponds to the fact that there are no faults on the test rig; namely, there are no false alarms in this experiment.

The distances, obtained on the basis of fault data in intershaft bearing under each condition, are compared with the corresponding radius of the sphere. As is shown in Figure 14, yellow cylinders which represent the distance are higher than the red cylinders representing the alarm threshold value. Thus, under every operating condition, this equipment is judged to be in a state of alarm. This analysis result corresponds to the fact that there are faults in intershaft bearing on the test rig; namely, no missing alarms occur in this experiment. 
TABLE 8: Cluster center and distance under normal and fault data.

\begin{tabular}{lcccc}
\hline \multicolumn{2}{c}{ Operating condition } & 1 & 2 & 3 \\
\hline \multirow{2}{*}{ Without fault } & Cluster center & {$[300,600,2.7864]$} & {$[897,1199,6.3217]$} & {$[1502,1803,9.0282]$} \\
& Distance & 0.0613 & 0.5346 & 0.3083 \\
\hline \multirow{2}{*}{ Fault } & Cluster center & {$[304,600,3.0273]$} & {$[901,1199,7.3804]$} & {$[1499,1800,9.0774]$} \\
& Distance & 3.7661 & 4.4019 & 3.5541 \\
\hline
\end{tabular}

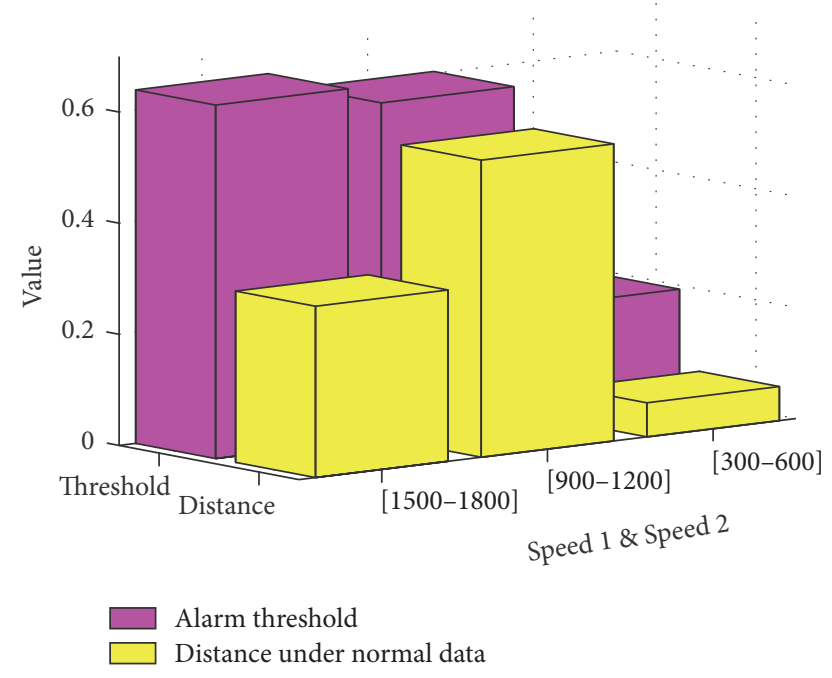

FIGURE 13: Alarm results for the normal data of the dual-rotor test rig.

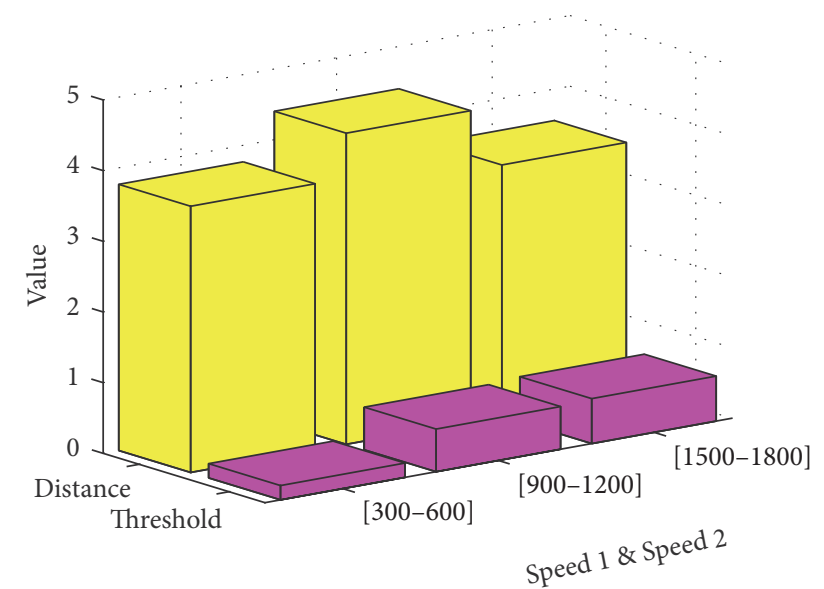

Alarm threshold

Distance under fault data

FIGURE 14: Alarm results for the fault data of the dual-rotor test rig.

By analyzing the above experimental data, it is obvious that the algorithm, proposed in this paper, is applicable to warning against faults early for dual-rotor equipment under time-varying operating conditions.

\section{Conclusions}

This paper proposes an early warning method based on SVDD and $K$-means for dual-rotor equipment under timevarying operating conditions. Vibration alarm thresholds suitable for different operating conditions can be decided by training SVDD with vibration data under each operating condition without faults. This alarm threshold can be expressed as several spheres whose different coordinates of center and radiuses are their controlling parameters. The cluster center of multigroup data, whose alarm states are to be determined, can be obtained through $K$-means algorithm. An early warning of vibration can be realized according to this relative position between the cluster center and the sphere. The proposed method, which combines the above two algorithms, has been tested through the vibration signals of a test rig for dual-rotor faults simulation. Consequently, it has been proved that the proposed method is valid on the early warning for dual-rotor equipment under time-varying conditions.

\section{Discussion}

There is a positive correlation between the speeds set and the load due to the special research object, and the load does not change when the speed set is constant. In other words, the speed factor considered in this paper can represent load. Therefore, only the influences of rotational speed set on the alarm threshold are studied in this paper.

If the present method will be used in other equipment (such as an electric motor whose speeds are the same but loads may be different), a parameter representing the load can be added to the speed set as an independent one easily. This can realize the early warning of comprehensive speed and the load parameters under time-varying operating conditions.

\section{Conflicts of Interest}

The authors declare no conflicts of interest.

\section{Acknowledgments}

Support for this research is provided by the National Key Technologies R\&D Program of China under Grant no. 2016YFF0203305 and the Fundamental Research Funds for the Central Universities under Grant no. JD1715. 


\section{References}

[1] H. R. Depold and J. Siegel, "Using diagnostics and prognostics to minimize the cost of ownership of gas turbines," in Proceedings of the 2006 ASME 51st Turbo Expo, pp. 845-851, May 2006.

[2] E. Hindle, R. Van Stone, C. Brogan, J. Vandike, K. Dale, and N. Gibson, "A prognostic and diagnostic approach to engine health management," in Proceedings of the 2006 ASME 51st Turbo Expo, pp. 673-680, May 2006.

[3] D. Balevic, "Heavy-duty gas turbine operating and maintenance considerations," GER, 2003.

[4] J. B. Ali, N. Fnaiech, L. Saidi, B. Chebel-Morello, and F. Fnaiech, "Application of empirical mode decomposition and artificial neural network for automatic bearing fault diagnosis based on vibration signals," Applied Acoustics, vol. 89, pp. 16-27, 2015.

[5] A. Widodo and B. Yang, "Support vector machine in machine condition monitoring and fault diagnosis," Mechanical Systems and Signal Processing, vol. 21, no. 6, pp. 2560-2574, 2007.

[6] V. N. Vapnik, The Nature of Statistical Learning Theory, Springer, 1995.

[7] B.-S. Yang, T. Han, and W.-W. Hwang, "Fault diagnosis of rotating machinery based on multi-class support vector machines," Journal of Mechanical Science and Technology, vol. 19, no. 3, pp. 846-859, 2005.

[8] I. Aydin, M. Karakose, and E. Akin, "A multi-objective artificial immune algorithm for parameter optimization in support vector machine," Applied Soft Computing, vol. 11, no. 1, pp. 120-129, 2011.

[9] S. Lee and K.-K. Seo, "Intelligent fault diagnosis based on a hybrid multi-class support vector machines and case-based reasoning approach," Journal of Computational and Theoretical Nanoscience, vol. 10, no. 8, pp. 1727-1734, 2013.

[10] Z. Yin and J. Hou, "Recent advances on SVM based fault diagnosis and process monitoring in complicated industrial processes," Neurocomputing, vol. 174, pp. 643-650, 2016.

[11] K. R. Fyfe and E. D. S. Munck, "Analysis of computed order tracking," Mechanical Systems and Signal Processing, vol. 11, no. 2, pp. 187-202, 1997.

[12] P. Borghesani, R. Ricci, S. Chatterton, and P. Pennacchi, "A new procedure for using envelope analysis for rolling element bearing diagnostics in variable operating conditions," Mechanical Systems and Signal Processing, vol. 38, no. 1, pp. 23-35, 2013.

[13] Z. Jiang, M. Hu, K. Feng, and Y. He, "Weak fault feature extraction scheme for intershaft bearings based on linear prediction and order tracking in the rotation speed difference domain," Applied Sciences-Basel, vol. 7, no. 937, 2017.

[14] G. Q. Ren, W.-C. Zhang, and B. Li, "Research on the gearbox fault signal relation to the sensitivity of variable working conditions," Applied Mechanics and Materials, vol. 494-495, pp. 921-924, 2014.

[15] Y. Gu, L. Song, T. Xu, L. Su, and G. Wu, "Research on Wind Turbine Gearbox Fault Warning Method under Variable Operational Condition," Acta Press, 2014.

[16] C. Lin and V. Makis, "Optimal Bayesian maintenance policy and early fault detection for a gearbox operating under varying load," Journal of Vibration and Control, vol. 22, no. 15, pp. 33123325, 2016.

[17] Y. Shao, K. M. Chris, J. Ou, and Y. Hu, "Gearbox deterioration detection under steady state, variable load, and variable speed conditions," Chinese Journal of Mechanical Engineering, vol. 22, no. 2, pp. 256-264, 2009.
[18] A. Kouadri, G. R. Ibrahim, and A. Albarbar, "Varying load detection in a gearbox system based on adaptive threshold estimation," in Proceedings of the International Conference on Mechanical, Manufacturing, Modeling and Mechatronics, IC4M 2016, Kuala Lumpur, Malaysia, February 2016.

[19] Z. Fu and A. Robles-Kelly, "On mixtures of linear svms for nonlinear classification," Joint Iapr International Workshop on Structural, Syntactic, and Statistical Pattern Recognition, vol. 5342, pp. 489-499, 2008.

[20] J. A. K. Suykens, J. De Brabanter, L. Lukas, and J. Vandewalle, "Weighted least squares support vector machines: robustness and sparce approximation," Neurocomputing, vol. 48, pp. 85$105,2002$.

[21] Q.-A. Tran, X. Li, and H. Duan, "Efficient performance estimate for one-class support vector machine," Pattern Recognition Letters, vol. 26, no. 8, pp. 1174-1182, 2005.

[22] B. Schölkopf, J. C. Platt, J. Shawe-Taylor, A. J. Smola, and R. C. Williamson, "Estimating the support of a high-dimensional distribution," Neural Computation, vol. 13, no. 7, pp. 1443-1471, 2001.

[23] D. M. J. Tax and R. P. W. Duin, "Support vector domain description," Pattern Recognition Letters, vol. 20, no. 11-13, pp. 1191-1199, 1999.

[24] Y. N. Can, "Modeling and fault prediction of complex system support vector machine," National Defense industry Press, 2015.

[25] O. L. Mangasarian and M. E. Thompson, "Chunking for massive nonlinear kernel classification," Optimization Methods \& Software, vol. 23, no. 3, pp. 365-374, 2008.

[26] K. R. Žalik, "An efficient $k$-means clustering algorithm," Pattern Recognition Letters, vol. 29, no. 9, pp. 1385-1391, 2008.

[27] A. K. Jain, “Data clustering: 50 years beyond K-means," Pattern Recognition Letters, vol. 31, no. 8, pp. 651-666, 2010.

[28] J. MacQueen, "Some methods for classification and analysis of multivariate observations," Proc. of Berkeley Symposium on Mathematical Statistics and Probability, pp. 281-297, 1967.

[29] V. S. Ananthanarayana, M. N. Murty, and D. K. Subramanian, "Efficient clustering of large data sets," Pattern Recognition, vol. 34, no. 12, pp. 2561-2563, 2001.

[30] A. Likas, N. Vlassis, and J. J. Verbeek, "The global k-means clustering algorithm," Pattern Recognition, vol. 36, no. 2, pp. 451-461, 2003.

[31] X. Xu, SVM Parameter Optimization and Its Application in the Classification [Ph.D. thesis], Dalian Maritime University, Dalian City, China, December 2014.

[32] X. Liu, D. Jia, and H. Li, "Research on Kernel Parameter Optimization of Support Vector Machine in Speaker Recognition," Science Technology and Engineering, vol. 10, no. 7, pp. 1669-1673, 2010.

[33] P. Chen, J. Wang, and H. Lee, "Model selection Of SVMs using GA approach," in Proceedings of the 2004 IEEE International Joint Conference on Neural Networks, Piscataway, NJ, USA, 2004.

[34] R. C. Eberhart and J. Kennedy, "A new optimizer using particle swarm theory," in Proceedings of the 6th International Symposium on Micro Machine and Human Science (MHS '95), Piscataway, NJ, USA, October 1995.

[35] T. Xiao, D. Ren, S. Lei et al., "Based on grid-search and PSO parameter optimization for Support Vector Machine," Intelligent Control and Automation, IEEE, 2015.

[36] D. W. Coit, "Genetic algorithms and engineering design," The Engineering Economist, vol. 43, no. 4, pp. 379-381, 1998. 
[37] D. E. Goldberg and J. H. Holland, "Genetic algorithms and machine learning," Machine Learning, vol. 3, no. 2-3, pp. 95-99, 1998.

[38] K. Muthulakshmi, R. M. Sasiraja, and V. Suresh Kumar, "The proper location and sizing of multiple distributed generators for maximizing voltage stability using PSO," Journal of Circuits, Systems and Computers, vol. 26, no. 4, Article ID 1750057, 2017.

[39] J. Kennedy and R. Eberhart, "Particle swarm optimization," in Proceedings of the IEEE International Conference on Neural Networks, pp. 1942-1948, Perth, Australia, December 1995.

[40] P. C. Bhat, H. B. Prosper, S. Sekmen, and C. Stewart, "Optimizing event selection with the random grid search," High Energy Physics-Phenomenology, 2017, High Energy PhysicsPhenomenology.

[41] "Siemens, LMS SCADAS [EB/OL]", 2017, https://www.plm .automation.siemens.com/zh/products/lms/testing//scadas/lab .shtml.

[42] ISO 10816-4:2009, Mechanical vibration, Evaluation of machine vibration by measurements on non-rotating. 


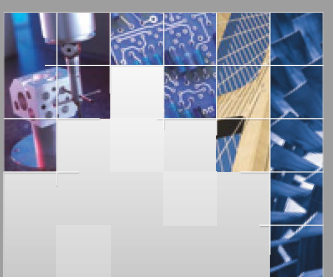

\section{Enfincering}
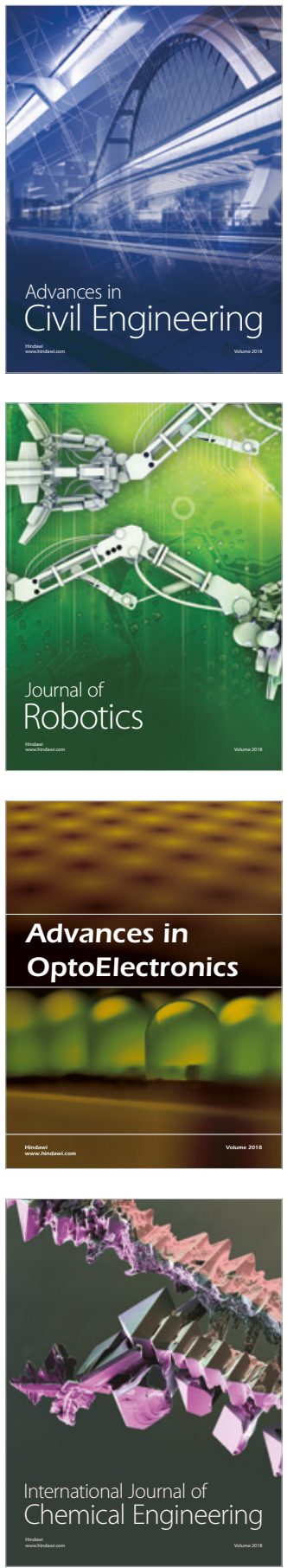

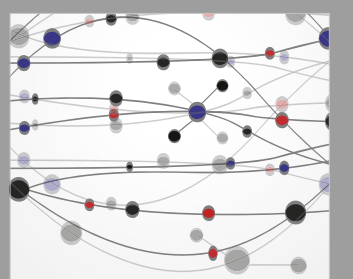

\section{Rotating \\ Machinery}

The Scientific World Journal

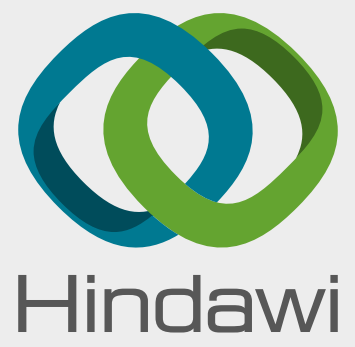

Submit your manuscripts at

www.hindawi.com
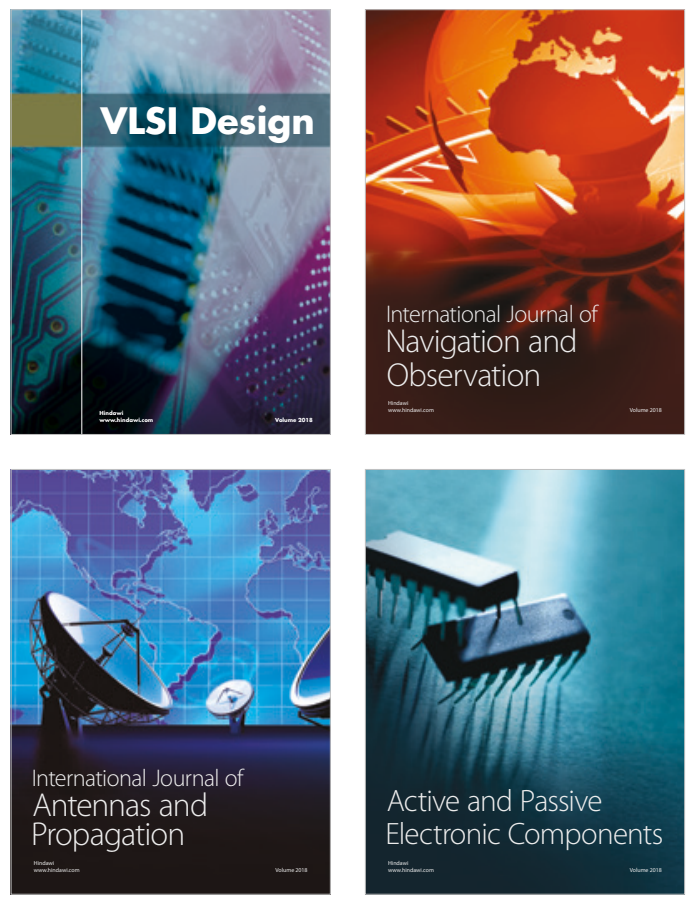
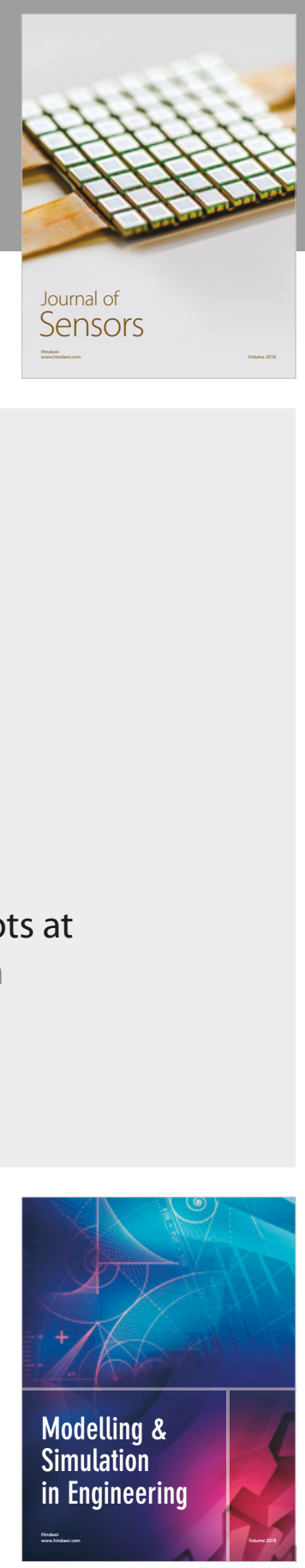

\section{Advances \\ Multimedia}
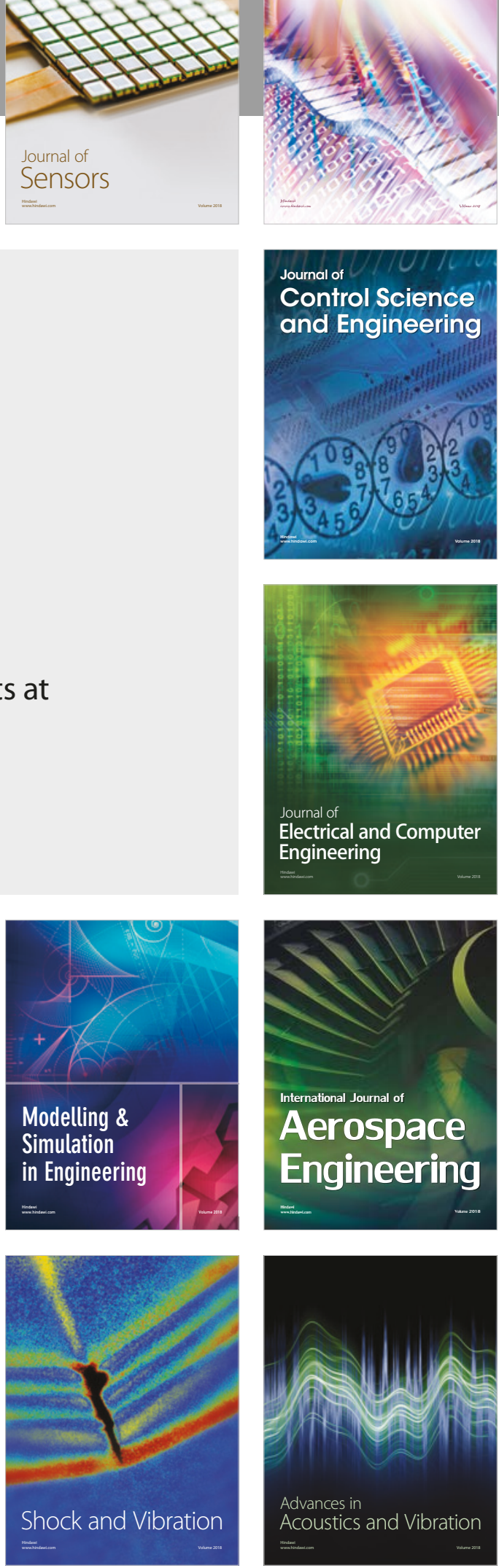